\title{
NOTE
}

\section{Synthesis of Aromatic Polyamide Having Fluorescein Moieties in the Main-Chain and Its Conversion into Ionically Cross-Linked Nanoparticles with $\mathrm{Ca}^{2+}$}

\author{
By Jun-ichi KADOKAWA, ${ }^{1, *}$ Takehiro NISHIKAWA, ${ }^{2}$ Yousuke SASAKI,${ }^{1}$ \\ Yuhki TANAKA, ${ }^{1}$ Takeshi KATO ${ }^{1}$ and Yoshiro KANEKO ${ }^{1}$
}

\section{KEY WORDS: Aromatic Polyamide / Fluorescein / Direct Polycondensation / Fluorescent Nanoparticles / Ion Chelater /}

In recent years, considerable interest has been devoted to developing new fluorescence dye-based probes as the excellent sensors for biomolecules, being sensitive, fast-responding, and capable of affording high spatial resolution via microscopic imaging. ${ }^{1}$ Among many fluorescence dyes, fluorescein is known to have a high quantum yield of fluorescence in aqueous solution and to be excitable at long wavelength. ${ }^{2}$ Over the years, a variety of fluorescein derivatives have been prepared, and used as laser dyes, fluorescent detection, and fluorophore for labeling and sensing biomolecules. ${ }^{3}$ Therefore, polymeric derivatives incorporating fluorescein moieties in the main-chains are expected as new photo-functional polymeric materials. ${ }^{4}$ For the purpose of the study on synthesis of such polymers, 5-aminofluorescien (1), a commercial reagent, can be considered to be a good starting compound. Because the compound has both amino and carboxylic acid groups in the case of the ring-opened form (quinoid form), its direct polycondensation possibly takes place under the appropriate conditions to give an aromatic polyamide having fluorescein moieties in the main-chain.

In this paper, accordingly, we report synthesis of the aromatic polyamide (2) having fluorescein moieties in the main-chain by the direct polycondensation of $\mathbf{1}$ using triphenyl phosphite/pyridine system as a condensing agent (Scheme 1). Furthermore, 2 was converted into ionically cross-linked nanoparticles by treatment with $\mathrm{CaCl}_{2}$ aqueous solution (Scheme 3).

\section{EXPERIMENTAL}

\section{Materials}

Monomer, 1 was purchased from Aldrich Chemical Co. Pyridine was purified by distillation. Other reagents and solvents were used without further purification.

\section{Direct Polycondensation of 1}

A typical example for the polycondensation was as follows (entry 2, Table I). Under argon, to a solution of $1(0.0521 \mathrm{~g}$, $0.150 \mathrm{mmol})$ in pyridine $(1.5 \mathrm{~mL})$, triphenyl phosphite

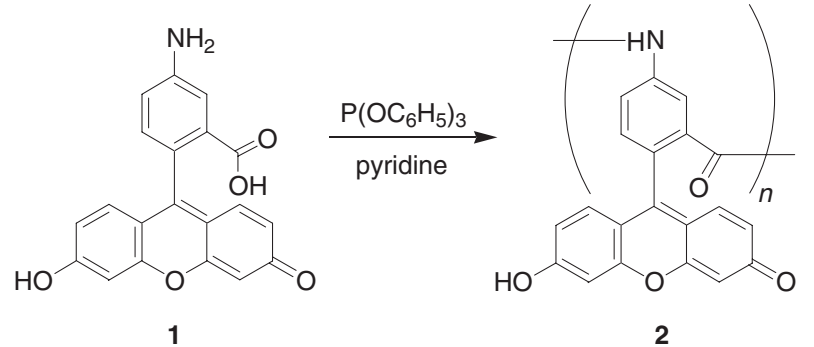

Scheme 1. Direct polycondensation of 1 using triphenyl phosphite/pyridine system as a condensing agent.

Table I. Direct polycondensation of 5-aminofluorescein using triphenyl phosphite/pyridine system as a condensing agent ${ }^{a}$

\begin{tabular}{cccccc}
\hline entry & $\begin{array}{c}\text { triphenyl phosphite } \\
\text { (equiv. for 1) }\end{array}$ & time (h) & yield (\%) & $M_{\mathrm{n}}{ }^{\mathrm{c}}$ & $M_{\mathrm{w}} / M_{\mathrm{n}}{ }^{\mathrm{c}}$ \\
\hline 1 & 1.27 & 24 & 68.2 & 3600 & 2.1 \\
2 & 2.56 & 24 & 63.0 & 2800 & 2.2 \\
3 & 12.7 & 24 & 51.6 & 1300 & 2.6 \\
4 & 12.7 & 48 & 62.7 & 2500 & 2.1 \\
5 & 1.27 & 24 & 36.4 & 3400 & 1.7 \\
& (diphenyl phosphite) & & & & \\
\hline
\end{tabular}

a Polycondensation was performed in pyridine at $100^{\circ} \mathrm{C}$ under argon ${ }^{b}$ Fraction insoluble in methanol, which was purified further by treatment with $1 \mathrm{~mol} / \mathrm{L} \mathrm{NaOH}$ aq. and $1 \mathrm{~mol} / \mathrm{L} \mathrm{HCl}$ aq. ${ }^{c}$ Determined by GPC measurement with $0.02 \mathrm{~mol} / \mathrm{L} \mathrm{LiCl} / \mathrm{DMF}$ as the eluent using poly(ethylene glycol) standards.

$(0.10 \mathrm{~mL}, 0.384 \mathrm{mmol})$ was added. After the mixture was stirred at $100{ }^{\circ} \mathrm{C}$ for $24 \mathrm{~h}$, the reaction mixture was poured into a large amount of methanol to precipitate the crude product. The precipitate was isolated by filtration and $1.0 \mathrm{~mol} / \mathrm{L} \mathrm{NaOH}$ aqueous solution was added to the obtained material. The insoluble fraction was separated by filtration and $1.0 \mathrm{~mol} / \mathrm{L}$ $\mathrm{HCl}$ aqueous solution was added to the filtrate. Thus, the obtained precipitate was isolated by filtration and dried under reduced pressure to give $2(0.0311 \mathrm{~g}, 0.0945 \mathrm{mmol})$ in $63.0 \%$ yield. ${ }^{1} \mathrm{H} \mathrm{NMR}\left(400 \mathrm{MHz}, 1.0 \mathrm{~mol} / \mathrm{L} \mathrm{NaOD} / \mathrm{D}_{2} \mathrm{O}\right) \delta 5.99$, 6.30, 6.63, 6.80, 7.04 (br, xanthene, 6H), 7.04, 7.20, 8.34, 8.45 (br, main-chain, 3H). IR (KBr) $1608.5 \mathrm{~cm}^{-1}(\mathrm{C}=\mathrm{O}$ of xanthene), $1670.2 \mathrm{~cm}^{-1}(\mathrm{C}=\mathrm{O}$ of amide $)$.

\footnotetext{
${ }^{1}$ Graduate School of Science and Engineering, Kagoshima University, Korimoto, Kagoshima 090-0065, Japan

${ }^{2}$ National Cardiovascular Center Research Institute, 5-7-1 Fujishirodai, Suita 565-8565, Japan

*To whom correspondence should be addressed (Tel: +81-99-285-7743, Fax: +81-99-285-3253, E-mail: kadokawa@eng.kagoshima-u.ac.jp).
} 


\section{Acetylation of 2}

Under argon, to a solution of $2(0.0093 \mathrm{~g}, 0.0282 \mathrm{mmol})$ in DMSO $(0.50 \mathrm{~mL})$, acetic anhydride $(0.20 \mathrm{~mL}, 2.12 \mathrm{mmol})$ was added and the mixture was stirred at $60^{\circ} \mathrm{C}$ for $18 \mathrm{~h}$. The reaction mixture was poured into a large amount water to precipitate the product. The precipitate was filtered, washed with chloroform, acetone, and methanol, and dried under reduced pressure to give $3(0.0070 \mathrm{~g}, 0.0189 \mathrm{mmol})$ in $79.2 \%$ yield. ${ }^{1} \mathrm{H}$ NMR (400 MHz, DMSO- $\left.d_{6}\right) \delta 1.91-2.33\left(\mathrm{br}, \mathrm{CH}_{3}\right.$, 3H), 6.15-7.21 (br, aromatics, 9H). IR (KBr) $1608.5 \mathrm{~cm}^{-1}$ $(\mathrm{C}=\mathrm{O}$ of xanthene $), 1678.0 \mathrm{~cm}^{-1}(\mathrm{C}=\mathrm{O}$ of amide $), 1762.8$ $\mathrm{cm}^{-1}(\mathrm{C}=\mathrm{O}$ of ester).

\section{Preparation of Fluorescent Nanoparticles}

An alkaline solution of $\mathbf{2}$ was prepared by mixing $\mathbf{2}$ (entry 2, Table I, $0.0070 \mathrm{~g}, 0.0021 \mathrm{mmol}$ ) with $1.0 \mathrm{~mol} / \mathrm{L} \mathrm{NaOH}$ aqueous solution $(21 \mu \mathrm{L}, 0.0021 \mathrm{mmol})$, followed by addition of water $(179 \mu \mathrm{L})$. The solution was slowly poured into $2.0 \mathrm{~mol} / \mathrm{L}$ $\mathrm{CaCl}_{2}$ aqueous solution to form a suspension. After filtration of the suspension, the filtrate was concentrated under reduced pressure until the precipitate was formed. The precipitate, which was separated by filtration, was dispersed in water with sonication and large aggregate was filtered off through membrane filter with pore size of $2 \mu \mathrm{m}$. The filtrate was evaporated under reduced pressure to give the ionically crosslinked material $(0.0040 \mathrm{~g})$. The material was dispersed in water to give the dispersion of fluorescent nanoparticles, which was subjected to fluorescence, SEM, and DLS measurements.

\section{Measurements}

NMR spectra were recorded on a JEOL ECX 400 spectrometer. IR spectra were recorded using a SHIMADZU FTIR-8400 spectrometer. GPC analyses were performed by using a HITACHI L-2130 with RI detector under the following conditions: Shodex K-803 and K-804 columns with 0.020 $\mathrm{mol} / \mathrm{L} \mathrm{LiCl} / \mathrm{DMF}$ as the eluent at a flow rate of $1.0 \mathrm{~mL} / \mathrm{min}$ at $60^{\circ} \mathrm{C}$. The calibration curve was obtained using poly(ethylene glycol) standards. TGA measurements were performed on a SII TG/DTA 6200 at a heating rate of $10^{\circ} \mathrm{C} / \mathrm{min}$. Fluorescence spectra were recorded on a Jasco FP-6300 fluorometer. Fluorescence quantum yields were calculated by using quinine sulfate in $0.10 \mathrm{~mol} / \mathrm{L}$ sulfuric acid as the reference absolute quantum efficiency $(\Phi=55 \%)$. The SEM images were obtained using a Hitachi S-4100 electron microscope. The DLS measurement was performed on a Zetasizer 3000 (Malvern Instruments).

\section{RESULTS AND DISCUSSION}

It has been reported that the triphenyl phosphite/pyridine system is one of good condensing agents for the direct polycondensation of amino benzoic acid derivatives. ${ }^{5,6}$ Therefore, we employed this condensing agent for the present study. A mixture of $\mathbf{1}$ and triphenyl phosphite in pyridine was heated at $100{ }^{\circ} \mathrm{C}$ for a desired time under argon. Then, the resulting mixture was poured into methanol to precipitate a crude

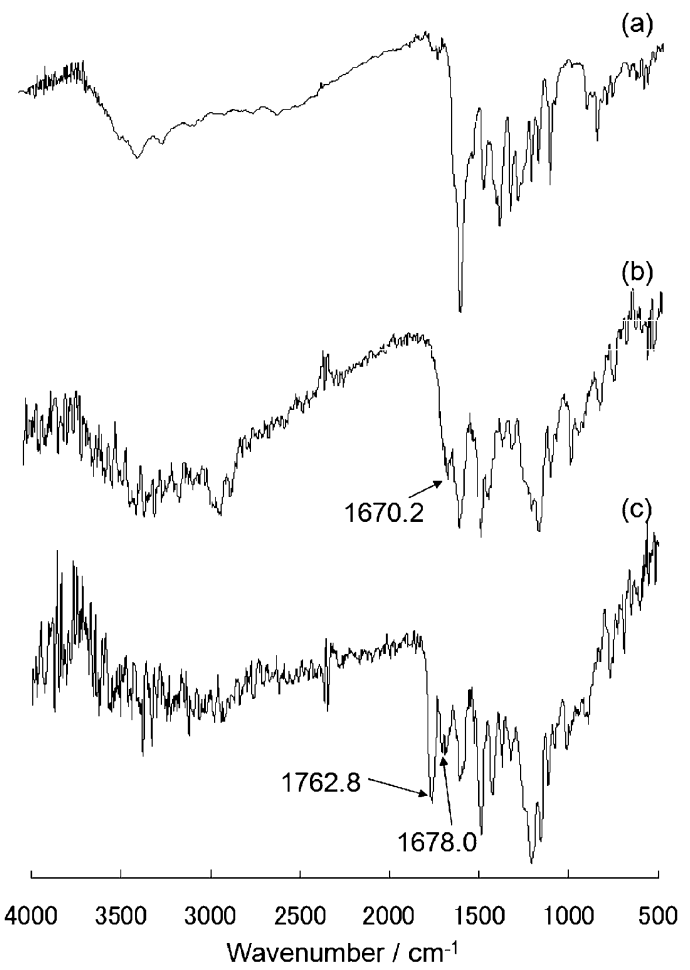

Figure 1. IR spectra of $\mathbf{1}$ (a), 2 (b), and $\mathbf{3}$ (c).

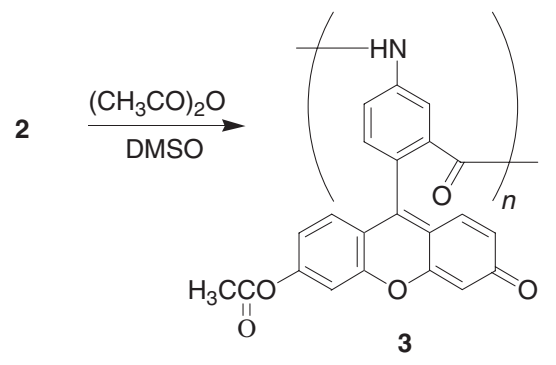

Scheme 2. Acetylation of 2.

product. The product was treated with $1.0 \mathrm{~mol} / \mathrm{L} \mathrm{NaOH}$ and the insoluble material was filtered off. After the filtrate was acidified, the obtained precipitate was isolated by filtration and dried under reduced pressure to give the polymeric product. The IR spectrum of the product in Figure $1 \mathrm{~b}$ exhibited a new absorption at $1670.2 \mathrm{~cm}^{-1}$ due to $\mathrm{C}=\mathrm{O}$ of amide linkage, which was not observed in that of $\mathbf{1}$ (Figure 1a). The ${ }^{1} \mathrm{H}$ NMR spectrum of the product $\left(1.0 \mathrm{~mol} / \mathrm{L} \mathrm{NaOD} / \mathrm{D}_{2} \mathrm{O}\right)$ showed aromatic signals due to both the main-chain at $\delta 6.9-8.5$ and the xanthene group at $\delta 5.9-7.0$. The above spectroscopic data fully supported the polyamide structure $\mathbf{2}$ of the product.

Although the GPC measurement of $\mathbf{2}$ was performed to estimate the molecular weight, the obtained chart did not show a clear elution curve, probably due to presence of the phenolic hydroxyl groups. Therefore, acetylation of $\mathbf{2}$ was conducted using acetic anhydride at $60^{\circ} \mathrm{C}$ (Scheme 2) and the product was isolated by precipitation of the reaction mixture into water, followed by washing with chloroform, acetone, and methanol to give the acetylated derivative. 


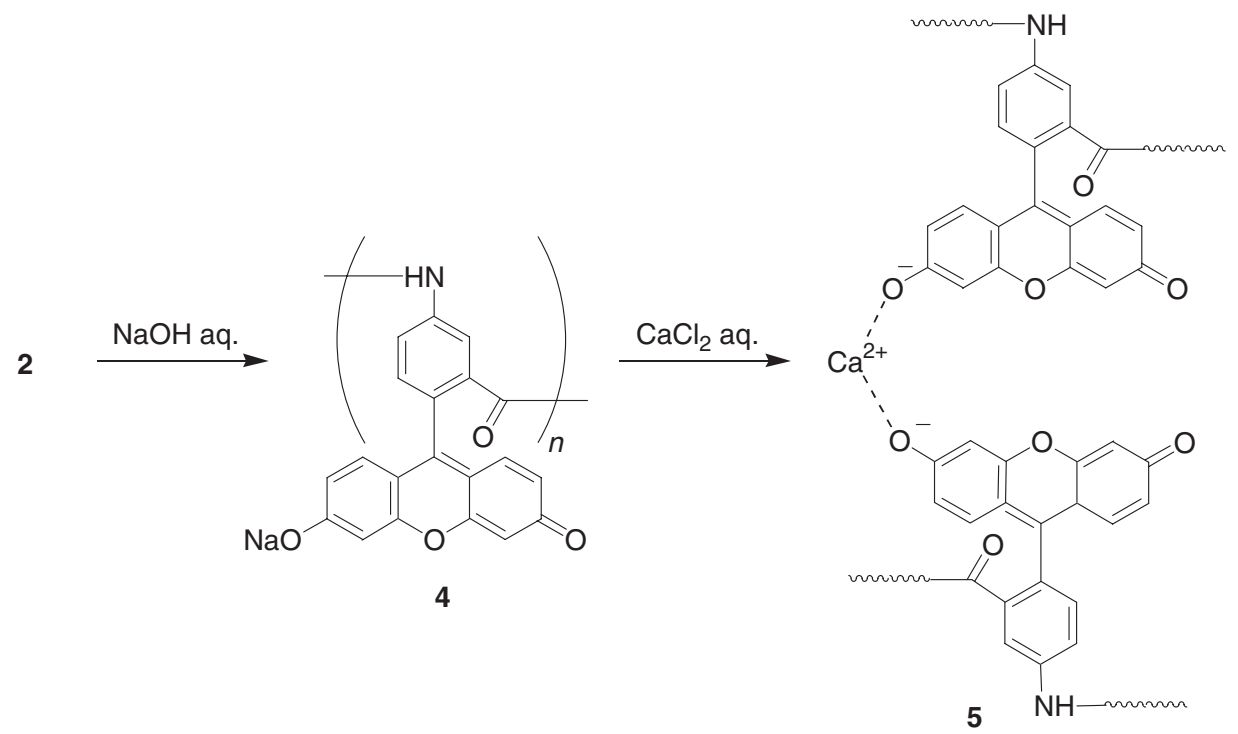

Scheme 3. Preparation of ionically cross-linked fluorescent nanoparticles.

The IR spectrum of the isolated material (Figure 1c) exhibited a new carbonyl absorption at $1762.8 \mathrm{~cm}^{-1}$ ascribable to ester linkage besides that at $1678.0 \mathrm{~cm}^{-1}$ due to amide linkage. Furthermore, the ${ }^{1} \mathrm{H}$ NMR spectrum of the same material in DMSO- $d_{6}$ showed the signal at around $\delta 2-2.3$ due to methyl protons of acetyl group besides the aromatic signals. The integrated ratio of former to latter was $c a$. 1:3. All the above data supported that the phenolic hydroxyl groups in $\mathbf{2}$ were fully acetylated to give $\mathbf{3}$. The acetylated derivative $\mathbf{3}$ was subjected to the GPC measurement with $0.02 \mathrm{~mol} / \mathrm{L} \mathrm{LiCl} /$ DMF as an eluent to estimate the value of molecular weight using poly(ethylene glycol) standards.

Table I shows the selected results of the polycondensation of 1 under some conditions. The yields of $c a$. 50-70\% were probably caused by incomplete conversion of $\mathbf{1}$ due to precipitation of the products during the polymerization. Use of higher ratios of triphenyl phosphite to $\mathbf{1}$ gave $\mathbf{2}$ with lower molecular weights when the polycondensation was performed under the condition of the same reaction time (entries 1-3). This is reasonably explained by as follows; the higher ratio of the condensing agent to the monomer provides the many reaction sites, resulting in a large number of polymer molecules with lower molecular weight. Indeed, longer reaction time using the same amount of triphenyl phosphite as entry 3 induces the further reaction between the polymers, leading to the higher molecular weight (entry 4). The similar tendency was observed in the previous studies on the direct polycondensation. ${ }^{7,8}$ There is a possibility for underestimation of the $M_{\mathrm{n}}$ values in Table I by the GPC analysis using poly(ethylene glycol) standards, because the $M_{\mathrm{n}}$ value of diacetylated fluorescein (molecular weight $=416$ ) was calculated as $c a$. 100 by the same GPC technique. When the polycondensation of 1 was performed under the same conditions as those of entry 1 using the condensing agent system consisting of diphenyl phosphite/pyridine, which had been used for direct polycondensation, ${ }^{9}$ the polyamide 2 with the similar $M_{\mathrm{n}}$ value was produced in lower yield (entry 5). Dichlorophenylphosphine/ $\mathrm{LiCl} /$ pyridine, one of the other previously reported condensing agent systems, ${ }^{10}$ gave an unknown product with low molecular weight.

The fluorescence spectrum of 2 excited at $495 \mathrm{~nm}$ in $0.1 \mathrm{~mol} / \mathrm{L} \mathrm{NaOH}$ aqueous solution showed an emission maximum peak at $517 \mathrm{~nm}$, which was typically attributed to the quinoid form of fluorescein by ring opening. This fluorescence pattern was same as that of $\mathbf{1}$ (excitation; $495 \mathrm{~nm}$, emission; $517 \mathrm{~nm}$ ) in $0.1 \mathrm{~mol} / \mathrm{L} \mathrm{NaOH}$ aqueous solution. A fluorescence quantum yield of $\mathbf{2}$ was calculated by using quinine sulfate as reference absolute quantum efficiency to be $22 \%$. This value was almost equal to that of a poly $(p-$ phenylene) having fluorescein moieties in the main-chain (20\%), which was synthesized in our previous study. ${ }^{4}$

It is well known that aromatic polyamides are thermally stable polymeric materials. ${ }^{11}$ To evaluate thermal stability of 2, TGA measurement was conducted. As shown in Figure 2, 2 underwent only $10 \%$ weight loss in the temperature range between 450 and $500{ }^{\circ} \mathrm{C}$, indicating good thermal stability of $\mathbf{2}$.

The $\mathrm{Ca}^{2+}$ mediated cross-linking of $\mathbf{2}$ (Scheme 3) was attempted to show formation of cross-linked material $\mathbf{5}$ because $\mathbf{2}$ had phenolic hydroxyl groups, that formed cross-linkages with divalent cations. An alkaline solution of $\mathbf{2}$ was gradually poured into $\mathrm{CaCl}_{2}$ aqueous solution to give a suspension. After an appropriate work-up procedure, the ionically cross-linked material 5 was isolated. The fluorescence spectrum of a dispersion of $\mathbf{5}$ in water $(0.40 \mathrm{mg} / \mathrm{mL})$ exhibited an emission maximum at $515 \mathrm{~nm}$ when excited at $495 \mathrm{~nm}$, which was a typical fluorescence peak of a ring-opened form of the fluorescein derivative. This fluorescence pattern was similar as that of $\mathbf{2}$ as aforementioned. However, an accurate quantum yield of $\mathbf{5}$ could not be obtained because its aqueous suspension did not exhibit clear absorption peaks of the fluorescein moiety 


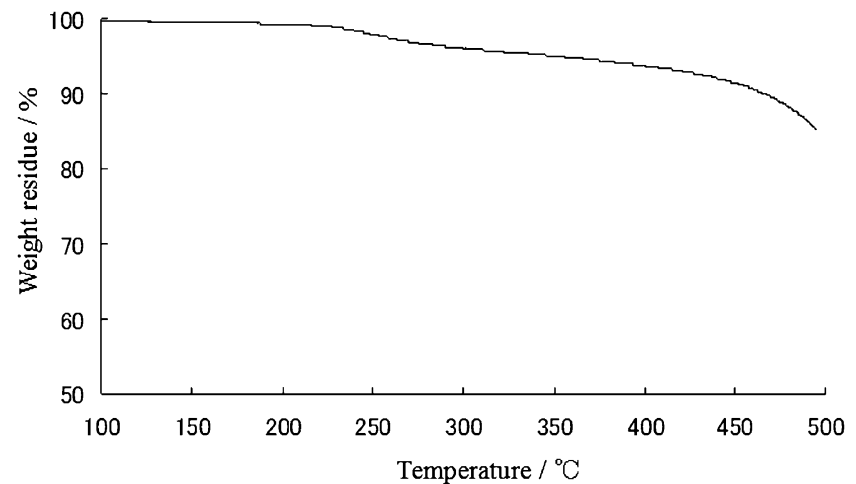

Figure 2. TGA trace of 2.

at around $500 \mathrm{~nm}$. This was probably attributed to the formation of nanoparticles of $\mathbf{5}$ in water. Figure 3 a shows the SEM image of the spin-coated sample from a dispersion of 5 in water. The nanoparticles with diameters of several tens $\mathrm{nm}$ were clearly seen in the image and their average diameter and standard deviation were calculated to be 50.8 and $9.15 \mathrm{~nm}$, respectively. The DLS measurement of a dispersion of $\mathbf{5}$ in water $(0.50 \mathrm{mg} / \mathrm{mL})$ in Figure $3 \mathrm{~b}$ also showed formation of nano-scale aggregates with mono modal profile (polydispersity index $=0.142$ ). The mean diameter was calculated to be $87.4 \mathrm{~nm}$, which was larger than that observed in the SEM image as described above. The difference was probably attributed to the different calculation methods for the average diameters in both the measurement, in which the average diameter in the DLS measurement was calculated from the intensity of scattering light, that was proportional to $\mathrm{d}^{6}$ (d: diameter of a nanoparticle), whereas number distribution was used for calculation of the average diameter of nanoparticles in the SEM measurement. The sample conditions (wet and dry) in these measurements might also affect the difference in particle size. The particle size decreased from $87.4 \mathrm{~nm}$ to $32.5 \mathrm{~nm}$ (Figure $3 b$ ) when $0.10 \mathrm{~mol} / \mathrm{L}$ EDTA aqueous solution was added to the suspension of the nanoparticles of $\mathbf{5}$ to obtain a nanoparticle suspension including $0.010 \mathrm{~mol} / \mathrm{L}$ EDTA. This suggests that the nanoparticles of 5 can be degraded by treating with $\mathrm{Ca}^{2+}$ ion chelator. ${ }^{12}$ The above fluorescence, SEM, and DLS results supported formation of the fluorescent nanoparticles by ionic cross-linking with $\mathrm{Ca}^{2+}$.

\section{CONCLUSIONS}

We reported the direct polycondensation of $\mathbf{1}$ using the condensing agent of triphenyl phosphite and pyridine to produce the aromatic polyamide $\mathbf{2}$ having fluorescein moieties in the main-chain. The IR and ${ }^{1} \mathrm{H}$ NMR spectra of the product fully supported the structure 2 . The molecular weights of the products, which were estimated by the GPC measurements of the acetylated derivative 3 , were affected by the reaction conditions such as the ratio of the condensing agent to the monomer and the reaction time. The polyamide $\mathbf{2}$ was further converted into the ionically cross-linked materials with

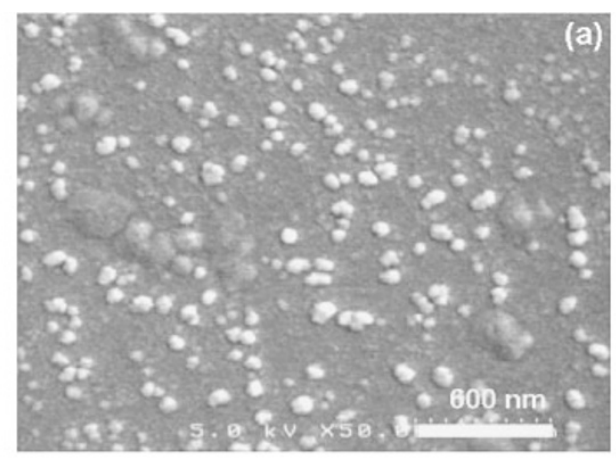

(b)

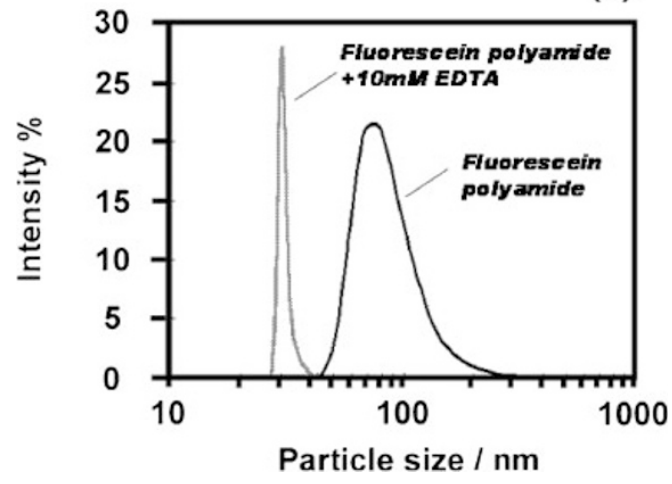

Figure 3. SEM image of the spin-coated sample, that was prepared from an aqueous suspension of $\mathbf{5}$ (a) and DLS profiles of dispersions of $\mathbf{5}$ $(0.50 \mathrm{mg} / \mathrm{mL})$ (b) before and after addition of EDTA aqueous solution.

divalent cations by treatment with $\mathrm{CaCl}_{2}$ aqueous solution. The SEM and DLS measurements of the product indicated formation of the nanoparticles with relatively low dispersity. Furthermore, the nanoparticles were degraded by treating with EDTA as a $\mathrm{Ca}^{2+}$ ion chelator. The fluorescence spectrum of a dispersion of the product in water exhibited a typical emission peak at $515 \mathrm{~nm}$ resulting from the excitation of fluorescein in the polyamide at $495 \mathrm{~nm}$.

Acknowledgment. T.N. thanks Dr. Tetsuji Yamaoka and Dr. Atsushi Mahara of the National Cardiovascular Center for DLS measurement.

Received: April 20, 2009

Accepted: June 25, 2009

Published: August 5, 2009

\section{REFERENCES}

1. R. A. Bissell, A. P. de Silva, H. Q. N. Gunarathe, P. L. M. Lynch, C. P. McCoy, G. E. M. Maguire, and K. R. A. S. Sandanayake, in "Fluorescent Chemosensors for Ion and Molecule Recognition," A. W. Czarnik, Ed., ACS Symposium Series 538, American Chemical Society, Washington DC, 1993, Chap. 9.

2. W. Sun, K. R. Gee, D. H. Klaubert, and R. P. Haugland, J. Org. Chem., 62, 6469 (1997).

3. "Handobook of Fluorescent Probes and Research Products", 9th ed., R. P. Haugland, Ed., Probes Inc., Eugene, 2002.

4. J. Kadokawa, M. Suenaga, and Y. Kaneko, Macromolecules, 41, 3750 
(2008).

5. N. Yamazaki, F. Higashi, and J. Kawabata, J. Polym. Sci., Part A: Polym. Chem., 12, 2149 (1974).

6. F. Higashi, J. Synth. Org. Chem. Jpn., 40, 922 (1982).

7. M. Ueda, M. Sato, and A. Mochizuki, Macromolecules, 18, 2723 (1985).

8. M. Ueda and M. Sato, Macromolecules, 20, 2675 (1987).
9. N. Yamazaki, J. Kawabata, and F. Higashi, J. Polym. Sci., Part A: Polym. Chem., 15, 1511 (1977).

10. F. Higashi, N. Kokubo, and M. Goto, J. Polym. Sci., Part A: Polym. Chem., 18, 2879 (1980).

11. J. Lin and D. C. Sherrington, Adv. Polym. Sci., 111, 177 (1994).

12. M. Kobašlija and D. T. McQuade, Biomacromolecules, 7, 2357 (2006). 